\title{
JACKSON KERNELS: A TOOL FOR ANALYSING THE DECAY OF EIGENVALUE SEQUENCES OF INTEGRAL OPERATORS ON THE SPHERE
}

\author{
T. Jordẽo And V. A. Menegatto
}

\begin{abstract}
Decay rates for the sequence of eigenvalues of positive and compact integral operators have been largely investigated for a long time in the literature. In this paper, the focus will be on positive integral operators acting on square integrable functions on the unit sphere and generated by a kernel satisfying a Hölder type assumption defined by average operators. In the approach to be presented here, the decay rate will be reached from convenient estimations on the eigenvalues of the operator themselves, with the help of specific properties of a generic approximation operator defined through the so-called generalized Jackson kernels. The decay rate has the same structure of those known to hold in the cases in which the Hölder condition is the classical one. Therefore, within the spherical setting, the abstract approach to be introduced here extends some classical results on the topic.
\end{abstract}

Mathematics subject classification (2010): 41A36, 45P05, 47A75, 47B34.

Keywords and phrases: Eigenvalue inequalities, eigenvalue decay, convolutions, integral operators, singular values, positive definiteness, Hölder inequality.

\section{REFERENCES}

[1] D. Azevedo And V. A. Menegatto, Eigenvalue decay of integral operators generated by power series-like kernels, Math. Inequal. Appl. 17 (2014), no. 2, 693-705.

[2] H. Berens, P. Butzer, AND S. PAWElKe, Limitierungsverfahren von Riehen mehrdimensionaler Kugelfunktionen und deren Saturationsverhalten, Publ. Res. Inst. Math. Sci. Ser. A 4 (1968/1969) 201-268.

[3] M. H. Castro, C. P. Oliveira and V. A. Menegatto, Laplace-Beltrami differentiability of positive definite kernels on the sphere, Acta Math. Sin. (Engl. Ser.) 29 (2013), no. 1, 93-104.

[4] F. СовOS AND T. KÜHN, Eigenvalues of integral operators with positive definite kernels satisfying integrated Hölder conditions over metric compacta, J. Approx. Theory 63 (1990), no. 1, 39-55.

[5] Feng Dai And YuAn XU, Approximation theory and harmonic analysis on spheres and balls, Springer Monographs in Mathematics, Springer, New York, 2013.

[6] C. M. Dikmen and J. B. Reade, Factorisation of positive definite operators, Arch. Math. (Basel) 91 (2008), no. 4, 339-343.

[7] Z. Ditzian And K. Runovskit, Averages on caps of $S^{d-1}$, J. Math. Anal. Appl. 248 (2000), no. 1, 260-274.

[8] J. C. Ferreira AND V. A. Menegatto, Eigenvalues of integral operators defined by smooth positive definite kernels, Integral Equations Operator Theory 64 (2009), no. 1, 61-81.

[9] G. B. Folland, Real analysis. Modern techniques and their applications, Second edition, Pure and Applied Mathematics (New York), A Wiley-Interscience Publication. John Wiley \& Sons, Inc., New York, 1999.

[10] W. FreEden, Constructive Approximation on the Sphere with Applications to Geomathematics, Claredon press, 1998.

[11] I. Gohberg, S. Goldberg, And N. KRUPnik, Traces and determinants of linear operators, Operator Theory: Advances and Applications, 116, Birkhäuser Verlag, Basel, 2000. 
[12] S. HEINRICH AND T. KÜHN, Embedding maps between Hölder spaces over metric compacta and eigenvalues of integral operators, Nederl. Akad. Wetensch. Indag. Math. 47 (1985), no. 1, 47-62.

[13] T. Jordẽo And V. A. Menegatto, Estimates for Fourier sums and eigenvalues of integral operators via multipliers on the sphere, Proc. Amer. Math. Soc., to appear.

[14] T. Jordão, V. A. Menegatto, And XingPing Sun, Eigenvalue sequences of positive integral operators and moduli of smoothness, In: Gregory E. Fasshauer; Larry L. Schumaker (Org.), Springer Proceedings in Mathematics \& Statistics - Approximation Theory XIV: San Antonio 2013. 83ed. New York: Springer, 2014, v. 83, p. 239-254.

[15] T. KÜHN, Eigenvalues of integral operators generated by positive definite Hölder continuous kernels on metric compacta, Nederl. Akad. Wetensch. Indag. Math. 49 (1987), no. 1, 51-61.

[16] T. KÜHN, Eigenvalues of integral operators with smooth positive definite kernels, Arch. Math. (Basel) 49 (1987), no. 6, 525-534.

[17] J. Levesley, Zuhua Luo, And XingPing Sun, Norm estimates of interpolation matrices and their inverses associated with strictly positive definite functions, Proc. Amer. Math. Soc. 127 (1999), no. 7, 2127-2134.

[18] P. I. LiZORKIN AND S. M. NiKOL'SKIǏ, A theorem concerning approximation on the sphere, Anal. Math. 9 (1983), no. 3, 207-221.

[19] M. Morimoto, Analytic functionals on the sphere, Translations of Mathematical Monographs, 178. American Mathematical Society, Providence, RI, 1998.

[20] A. Pietsch, Eigenvalues and s-numbers, Cambridge Studies in Advanced Mathematics, 13. Cambridge University Press, Cambridge, 1987.

[21] S. S. Platonov, On some problems in the theory of the approximation of functions on compact homogeneous manifolds, (Russian) Mat. Sb. 200 (2009), no. 6, 67-108; translation in Sb. Math. 200 (2009), no. 5-6, 845-885.

[22] J. B. Reade, Eigenvalues of Lipschitz kernels, Math. Proc. Cambridge Philos. Soc. 93 (1983), no. 1, $135-140$.

[23] J. B. READE, Eigenvalues of positive definite kernels, SIAM J. Math. Anal. 14 (1983), no. 1, 152-157.

[24] R. SCHABACK, A unified theory of radial basis functions, Native Hilbert spaces for radial basis functions II. Numerical analysis in the 20th century, Vol. I, Approximation theory, J. Comput. Appl. Math. 121 (2000), no. 1-2, 165-177.

[25] M. SCHREINER, Locally supported kernels for spherical spline interpolation, J. Approx. Theory 89 (1997), no. 2, 172-194.

[26] G. Szegö, Orthogonal polynomials, Fourth edition, American Mathematical Society, Colloquium Publications, Vol. XXIII. American Mathematical Society, Providence, R. I., 1975. 